\title{
BMJ Open Return to work of cancer patients after a multidisciplinary intervention including occupational counselling and physical exercise in cancer patients: a prospective study in the Netherlands
}

\author{
Monique C J Leensen, ${ }^{1}$ Iris F Groeneveld, ${ }^{2}$ Iris van der Heide, ${ }^{1}$ Tomas Rejda, ${ }^{3}$ \\ Peter L J van Veldhoven, ${ }^{4}$ Sietske van Berkel, ${ }^{5}$ Aernout Snoek, ${ }^{5}$ \\ Wim van Harten, ${ }^{6,7,8}$ Monique H W Frings-Dresen, ${ }^{1}$ Angela G E M de Boer ${ }^{1}$
}

To cite: Leensen MCJ, Groeneveld IF, Heide Ivder, et al. Return to work of cancer patients after a multidisciplinary intervention including occupational counselling and physical exercise in cancer patients: a prospective study in the Netherlands. BMJ Open 2017;7:e014746. doi:10.1136/ bmjopen-2016-014746

- Prepublication history and additional material are available. To view these files please visit the journal online (http://dx.doi. org/10.1136/bmjopen-2016014746).

Received 14 0ctober 2016 Revised 31 January 2017 Accepted 4 April 2017

CrossMark

For numbered affiliations see end of article.

Correspondence to

Dr Angela G E M de Boer; a.g. deboer@amc.nl

\section{ABSTRACT}

Objectives To support return to work (RTW) among cancer patients, a multidisciplinary rehabilitation programme was developed which combined occupational counselling with a supervised physical exercise programme during chemotherapy. The aim was to investigate RTW rates of cancer patients and to evaluate changes in work-related quality of life and physical outcomes.

Design Longitudinal prospective intervention study using a one-group design.

Setting Two hospitals in the Netherlands.

Participants of the eligible patients, $56 \%$ participated; 93 patients with a primary diagnosis of cancer receiving chemotherapy and on sick leave were included. Patients completed questionnaires on RTW, the importance of work, work ability (WAI), RTW self-efficacy, fatigue (MFI), and quality of life (EORTC QLQ C-30) at baseline and 6,12 and 18 months follow-up. Before and after the exercise programme 1-repetition maximum (1RM) muscle strength and cardiorespiratory fitness $\left(\mathrm{VO}_{2}\right.$ peak) were assessed.

Results Six months after the start of a multidisciplinary rehabilitation programme that combined occupational counselling with a supervised physical exercise programme, $59 \%$ of the cancer patients returned to work, $86 \%$ at 12 months and $83 \%$ at 18 months. In addition, significant improvements $(p<0.05)$ in the importance of work, work ability, RTW self-efficacy, and quality of life were observed, whereas fatigue levels were significantly reduced. After completing the exercise programme, 1RM muscle strength was significantly increased but there was no improvement in $\mathrm{VO}_{2}$ peak level.

Conclusions RTW rates of cancer patients were high after completion of the multidisciplinary rehabilitation programme. A multidisciplinary rehabilitation programme which combines occupational counselling with a supervised physical exercise programme is likely to result in RTW, reduced fatigue and increased importance of work, work ability, and quality of life.

\section{Strengths and limitations of the study}

- This is the first study to combine a physical training programme with occupational counselling in a multidisciplinary rehabilitation programme.

- Validated questionnaires were used to assess work ability, work limitations, self-efficacy, fatigue, and quality of life.

Because of the lack of a control group, there is no direct proof of the effects of our multidisciplinary programme.

- Possible biases are in the selection of participants with respect to the type of cancer, the high number of females (84\%), and the relatively high level of education.

- The cancer patients who were included were highly motivated to participate while those who did not participate might have been less motivated and more likely to have a different lifestyle.

\section{INTRODUCTION}

Survival rates of cancer have been rising over due to early detection and improved treatment. On the other hand treatment for cancer may have detrimental effects, as many cancer survivors experience long-term negative physical and/or psychological effects from cancer or cancer-related treatment. A large proportion of cancer patients stop working after diagnosis ${ }^{1}$ because of the intensity of the treatment regimen or the prognosis. ${ }^{2}$ When treatment has ended, the majority of cancer survivors will attempt to return to work, as being employed is synonymous with a normal life ${ }^{3}$ and is regarded as a marker of complete recovery. ${ }^{4}$ Moreover, employment provides a sense of structure, income and identity, and a revival of social contacts. ${ }^{56}$ 
However, cancer survivors experience return to work (RTW) difficulties. Eighteen months after diagnosis, a third of all cancer survivors did not succeed in returning to work $^{7}$ and overall cancer survivors have 1.4 times higher risk of being unemployed compared with healthy controls. ${ }^{8}$ When work is resumed, working hours, work ability, and work functioning may be affected as well. ${ }^{79}$ Therefore, it is important to provide employed cancer patients with interventions or rehabilitation programmes to support their work resumption.

Interventions to enhance work resumption should address multiple factors because rehabilitation strategies that target both health-related and work-related factors may be effective in enhancing work resumption and subsequent work performance. ${ }^{10}$ This was shown in a Cochrane review reporting modest evidence for the effectiveness of multidisciplinary interventions which incorporated physical, psychological and vocational components. ${ }^{11}$

A physical exercise programme may attenuate the side effects of chemotherapy such as reduced muscle strength and cardiorespiratory fitness, fatigue, and physical limitations. Findings from previous reviews and meta-analyses show that physical exercise of moderate to high intensity has beneficial effects on cardiorespiratory fitness, muscle strength, cancer-related fatigue, and quality of life, both during and following treatment. ${ }^{12-14}$ Moreover, the improvement of physical functioning and the reduction of fatigue may facilitate RTW. Cancer survivors perceived that participating in a physical exercise programme improved their fitness and confidence in physical abilities, and provided them with renewed energy that led to less fatigue and being more physically active in daily life. ${ }^{15}$ According to the interviewed survivors, these effects may have facilitated their RTW. Moreover, controlled effect studies found a beneficial effect of an exercise intervention on RTW rates and the number of working hours. ${ }^{1416}$

Rehabilitation that focuses on work may enhance RTW in cancer survivors as well. Work-related factors such as diminished work demands and provision of accommodation at the workplace significantly improve work outcomes in this population. ${ }^{17} 18$ Adequate vocational guidance by an occupational physician (OP) might be another strategy to facilitate RTW of cancer patients. ${ }^{19} 20$ An OP can provide advice to counteract perceived barriers and limitations in work, for instance by making adjustments in work tasks and/or work hours, by recruiting social support from the workplace, and by setting up gradual work resumption. ${ }^{21}$ In addition to this practical support, an OP may help to improve the attitude towards RTW or address misconceptions about work ability. Negative expectations about illness, future work capacity and RTW are associated with slower RTW in patients with different types of chronic disorders. ${ }^{22} 23$ Also, personal factors such as self-assessed work ability ${ }^{24}$ or motivational factors such as the intention to RTW or meaning of work, ${ }^{7}$ that may affect RTW, can be addressed by an OP.
Advice and guidance provided by an OP, given at an early stage, combined with a physical exercise programme, may increase the likelihood of a timely and enduring RTW in cancer patients. Hence, a multidisciplinary rehabilitation programme was developed to provide support regarding RTW early in the treatment process, consisting of both exercise training and specialised occupational counselling. ${ }^{21}$ In this multidisciplinary rehabilitation, counselling was provided by an oncological OP (OOP), who is an OP specialised in supporting patients with cancer experiencing work-related problems and who works within the clinical setting.

The aim of this study is to investigate RTW for cancer patients who completed this multidisciplinary rehabilitation programme and to evaluate changes in work ability, self-efficacy, work limitations, muscle strength, physical fitness, fatigue levels and quality of life before and after programme completion.

\section{METHODS}

\section{Study design and population}

This prospective study using a one-group design was conducted between August 2011 and February 2015 in Haaglanden Medical Centre (HMC) in The Hague and Isala in Zwolle, two large medical centres in the Netherlands. Eligible patients were aged between 18 and 60 years, had a primary diagnosis of cancer, and were being treated with chemotherapy with curative intent. In addition, they had been in paid employment at the time of diagnosis, were absent from work or intended to report sick before the start of treatment, and were able to complete a questionnaire in Dutch. Patients with severe mental disabilities or those who were physically unable to perform exercise training were excluded from participation, as were patients with testicular cancer, as the latter group experience relatively few problems in regard to RTW. $^{82526}$

\section{Procedure}

Patients from these hospitals were invited to participate in the study by their treating oncologist and were consecutively assigned to the multidisciplinary intervention if they met the eligibility criteria for the study. During the first 4 months of our study, registration records on the eligibility of newly-diagnosed patients, their willingness to participate, and reasons for non-participation were completed by the oncologists and oncology nurses.

Informed consent was obtained from all individual participants included in the study. The intervention started immediately after inclusion and had a duration of approximately 4 months.

Participants received four questionnaires; at entry into the programme, and 6 (T1), 12 (T2) and 18 months (T3) later to obtain information on their RTW, sickness leave duration, importance of work, work ability, work limitations, fatigue level, quality of life, self-efficacy concerning RTW, and physical activity level. Data 
on sociodemographics, cancer-related and work-related factors were collected at baseline. The study protocol was approved by the hospitals' medical ethics committees (ref no 2011_099). The study has been reported according to the STROBE checklist for cohort studies (see supplementary files).

\section{Multidisciplinary rehabilitation programme}

The multidisciplinary rehabilitation programme consisted of personal counselling on work-related issues by an OOP and supervised physical exercise in a clinical setting during curative treatment. The intervention protocol has been described in detail in a previous paper. ${ }^{21}$

Before the exercise programme started, participants underwent a sports medical assessment by a sports physician in order to: (1) assess their cardiorespiratory fitness by measuring peak oxygen consumption $\left(\mathrm{VO}_{2}\right.$ peak) during an incremental exercise test (ramp) on a bicycle until exhaustion; and (2) trace possible physical contraindications for training. Within 2 weeks of the sports medical assessment, participants embarked on a 12-week, twice-weekly physical training programme, supervised by a trained physiotherapist. The moderate-to-vigorous intensity exercise sessions lasted 1 hour and included interval training on a cycle ergometer and resistance exercises of the large muscle groups. The intensity of the exercises was set individually and expressed as a percentage of the maximal workload, assessed by a steep-ramp test in the case of interval training, ${ }^{27}$ or as a percentage of the 1-repetition maximum (1-RM) for strength exercises. ${ }^{12}$ Exercises were performed ranging from two series of eight repetitions to three series of 12 repetitions with increasing weight. Muscle groups were tested with strength equipment that was also used for the resistance training, performing leg pressand deltoid pulley. After completion of the exercise programme, muscle strength and $\mathrm{VO}_{2}$ peak were tested again.

Alongside the exercise programme, participants received 1-3 individual counselling sessions conducted by an OOP. Following a structured protocol, various work-related topics were discussed. The OOP provided advice on gradual work resumption, taking into account a patient's job type, medical situation, own opinions, and work ability. All participants had a first counselling session scheduled early during the intervention programme. Depending on the patient's preferences and the extent to which he or she was experiencing barriers to work, a second and third counselling session was planned.

\section{Measurements}

Sociodemographic variables, clinical characteristics and workrelated factors

The sociodemographic variables measured at baseline included age and gender of the participants, their education level, and marital status. Clinical characteristics were diagnosis (categorised into breast cancer, colorectal cancer or non-Hodgkin's lymphoma), treatment modalities additional to chemotherapy (surgery, radiotherapy and/or hormonal treatment), and time since diagnosis. Work-related factors assessed in the baseline questionnaire were working hours, type of contract (permanent, temporary or self-employed), job tenure in years in current position, breadwinner status (sole, shared or no), shift work (no shift work, irregular service, shift work), company size (small (1-10), medium (10-100), large $(>100))$, and date of sick leave.

\section{RTW outcome measures}

Primary outcome RTW is defined as any work resumption, irrespective of the number of hours a participant worked before diagnosis. Participants were asked at 6, 12 and 18 months follow-up whether they had returned to work, and if so on which date. In addition, time to RTW after sick leave was calculated as the number of days between the first date of sick leave and the first day of work resumption.

\section{Secondary outcome measures}

Secondary outcomes included perceptions regarding work, such as the importance of work, work ability, self-efficacy regarding RTW and work limitations; physical factors, such as muscle strength, cardiorespiratory fitness, physical activity level and fatigue; and quality of life.

Importance of work was measured by rating the perceived importance of work in one's current situation on a visual analogue scale (VAS) ranging from 0 to 10, with higher scores indicating higher importance of work. This measure gains insight into the meaning of work as experienced by the cancer patients at the time of measurement. Work ability was assessed with the first item of the Work Ability Index (WAI), ${ }^{28}$ which measured current work ability compared with the lifetime best on a scale from 0 to 10 (higher is better). Self-efficacy regarding RTW was measured using the 11-item self-efficacy scale developed by Lagerveld $e t a l^{29}$ with higher scores indicating higher self-efficacy. Work limitations were assessed using the Work Limitations Questionnaire (WLQ) ${ }^{30}$ The WLQ asks participants to rate their difficulty to perform 25 specific job demands in the last 2 weeks and contains four work limitation scales that report the degree to which health problems interfere with job demands, expressed as a percentage of time $(0 \%$ limited none of the time $-100 \%$ limited all of the time).

Muscle strength and cardiorespiratory fitness were assessed during measurements before and after the exercise programme as the results of 1-RM tests for muscle strength, and exercise testing using both the steep ramp test (expressed as Watts (W)) and $\mathrm{VO}_{2}$ peak test. ${ }^{12}{ }^{31}$ Fatigue was measured with the Multidimensional Fatigue Inventory (MFI), a 20-item instrument designed to measure fatigue in five dimensions. ${ }^{32}$ The corresponding subscales range from 4 to 20 , in which higher scores indicate more fatigue. Physical activity level was obtained by calculating the average amount of hours a participant had performed activities of moderate to high intensity in work, household, leisure time, or sports using questions from 
the short questionnaire to enhance health enhancing physical activity (SQUASH). ${ }^{33}$

Health-related quality of life (HRQoL) was assessed using the EORTC-QLQ-C30, specifically designed for cancer patients. ${ }^{34}$ This 30 -item list incorporates five functional scales (physical, role, emotional, cognitive and social functioning), three symptom scales (fatigue, nausea and vomiting, and pain), six single items on symptoms, and one overall quality of life scale. Scales and items ranged from 0 to 100 ; higher scores on the functional scales indicated better quality of life, whereas higher scores on the symptom scales and items represented worse quality of life. Scores on all different scales were calculated according to their published scoring algorithms. For scales containing missing values, the halfscale imputation rule was applied which is the most used procedure in the published scoring algorithms.

\section{Statistical analysis}

Data were analysed using IBM SPSS Statistics version 22, and R software (R Foundation 2008, from http://www. $\mathrm{R}$-project.org). Descriptive statistics were used to characterise the study population and to display outcomes measured at baseline and follow-up measurements. Data on eligibility and willingness to participate from the first 4 months of the study were extrapolated to the total study period. To assess the association between adherence to the physical exercise programme and the number of counselling sessions, we assessed a Spearman's rho correlation. The rate of return to work over time was evaluated using generalised estimating equation analyses with identity covariance structure and using a binomial distribution with a logit link. Time was included in the model as a fixed effect, and a random effect for subject was incorporated. This analysis adjusts for the non-independence of observations over time and handles missing values. The median time until RTW was analysed with a Kaplan-Meier survival analyses.

The secondary outcomes measured at 6, 12 and 18 months after inclusion into the study were analysed with the Skillings-Mack test to examine any change in scores over time. The Skillings-Mack test is an adaptation of the non-parametric Friedman test, that can be used to test repeated measurements in case of non-normal distributed data, which handles missing data that were acquired due to non-response. In case of a statistically significant main effect of time, post hoc analyses with Bonferroni corrections for multiple comparisons were performed by exact Wilcoxon signed rank tests. To avoid performing a great range of comparisons, these analyses were performed for both the difference between baseline and $\mathrm{T} 1$, to assess changes during rehabilitation, and between $\mathrm{T} 1$ and $\mathrm{T} 3$ to assess changes over the remaining follow-up period.

Muscle strength and cardiorespiratory fitness were measured before and after programme completion. Differences between these measurements were assessed by a paired Student's t-test. Of all pairwise comparisons, mean differences including $95 \%$ CI were calculated. A value of $\mathrm{p}<0.05$ was considered statistically significant.

\section{RESULTS}

\section{Study population}

Of the patients treated at the oncology departments of the participating hospitals, $49 \%$ were not eligible for participation, mostly because they were older than 60 years. Regarding the eligible patients, $56 \%$ were willing to participate in this study. There was no difference between participants and non-participants in gender and age at baseline. Of the 95 included patients who had completed the rehabilitation programme, two participants did not return a baseline questionnaire and were excluded. This finally resulted in a study population of 93 patients. The second questionnaire at 6 months follow-up was completed by 82 of the 93 participating patients (response rate of $88 \%$ ). At 12 months of follow-up, the questionnaire was returned by 72 of the 93 participants $(77 \%)$ and at 18 months of follow-up, 68 of the 93 participants returned the questionnaire $(73 \%)$. The adherence rate to the exercise programme was $86 \%$ and on average participants attended 20.5 of the 24 planned exercise sessions. Regarding the occupational counselling sessions, 94\% of the participants attended the first protocoled session, $35 \%$ attended the optional second sessions, and $8 \%$ had a third session with the OOP. Reasons for not attending the second or third sessions included: already returned to work ( $n=27)$; receiving good support from the company's occupational physician $(n=25)$; receiving good support from their employer $(n=18)$; and not considering further support necessary $(\mathrm{n}=10)$.

The Spearman correlation between the adherence to the exercise programme and the number of counselling sessions was 0.22 .

The baseline sociodemographics, work- and disease-related characteristics of the study population are presented in table 1. Participants had a mean age of 48 years and $90 \%$ was female. The majority of the participants had breast cancer $(84 \%)$; other cancer diagnoses were colorectal cancer and non-Hodgkin's lymphoma. Approximately $89 \%$ underwent surgery, and $76 \%$ received radiotherapy and/or hormonal treatment.

Before diagnosis and treatment, participants worked on average 28 hours per week. The majority of participants worked at a large company of more than 100 employees, were shared breadwinners, and did not perform any shift work.

\section{Return to work}

The primary outcome was the rate of RTW: the percentage of participants who returned to work at T1, T2 and T3. The rate of RTW increased significantly over time $(\mathrm{F}=8.51, \mathrm{p}<0.001)$. Six months after inclusion in the study, 49 participants $(59 \%)$ had, at least partially, returned to work. The rate of RTW increased to $86 \%$ of the study population at 12 months follow-up, and this percentage 


\begin{tabular}{|c|c|c|}
\hline \multicolumn{2}{|l|}{ Characteristic } & $\begin{array}{l}\text { Participants } \\
(n=93)\end{array}$ \\
\hline \multicolumn{3}{|c|}{ Sociodemographics } \\
\hline \multicolumn{2}{|c|}{ Gender (\% female, (n)) } & $90.3 \%(84)$ \\
\hline \multicolumn{2}{|c|}{ Age (years; mean $( \pm S D)$ ) } & $47.9(7.4)$ \\
\hline \multirow{3}{*}{$\begin{array}{l}\text { Education level } \\
(\%(n))\end{array}$} & Low & $14.0 \%(13)$ \\
\hline & Intermediate & $33.3 \%(31)$ \\
\hline & High & $52.7 \%(49)$ \\
\hline \multirow{3}{*}{$\begin{array}{l}\text { Marital status } \\
(\%(n))\end{array}$} & Single & $19.4 \%(18)$ \\
\hline & Not living with partner & $9.7 \%(9)$ \\
\hline & Living with partner & $71.0 \%(66)$ \\
\hline \multicolumn{3}{|c|}{ Disease-related variables } \\
\hline \multirow{4}{*}{$\begin{array}{l}\text { Cancer type (\% } \\
\text { (n)) }\end{array}$} & Breast & $83.9 \%(78)$ \\
\hline & Colorectal & $8.6 \%(8)$ \\
\hline & Non-Hodgkin's lymphoma & $5.4 \%(5)$ \\
\hline & Other & $2.2 \%(2)$ \\
\hline \multicolumn{2}{|c|}{ Days since diagnosis (mean $( \pm S D)$ ) } & $61.9(44.3)$ \\
\hline \multicolumn{2}{|c|}{ Type of chemotherapy (\% adjuvant $(n))$} & $87.1 \%(81)$ \\
\hline \multirow{4}{*}{$\begin{array}{l}\text { Treatment } \\
\text { additional to } \\
\text { chemotherapy } \\
(\% \text { (n)) }\end{array}$} & Surgery & $89.2 \%(83)$ \\
\hline & Radiotherapy & $30.1 \%(28)$ \\
\hline & Hormone treatment & $12.9 \%(12)$ \\
\hline & $\begin{array}{l}\text { Radiotherapy + hormone } \\
\text { treatment }\end{array}$ & $33.3 \%(31)$ \\
\hline \multirow{3}{*}{$\begin{array}{l}\text { Type of contract } \\
(\%(n))\end{array}$} & Permanent employment & $78.5 \%(73)$ \\
\hline & Temporary employment & $5.4 \%(5)$ \\
\hline & Self-employed & $11.8 \%(11)$ \\
\hline \multicolumn{3}{|c|}{ Work-related variables } \\
\hline \multicolumn{2}{|c|}{ Weekly working hours (mean $( \pm S D)$ ) } & $28.3(10.3)$ \\
\hline \multicolumn{2}{|c|}{ Years in current employment } & $10.8(8.4)$ \\
\hline \multicolumn{2}{|c|}{ Years in paid employment } & $22.9(9.6)$ \\
\hline \multirow[t]{3}{*}{ Company size } & Large $(>100)$ & $62.5 \%(55)$ \\
\hline & Medium (10-99) & $22.7 \%(20)$ \\
\hline & Small (1-9) & $9.1 \%(8)$ \\
\hline \multirow[t]{3}{*}{ Shift work } & No shiftwork & $81.3 \%(74)$ \\
\hline & Irregular service & $16.5 \%(15)$ \\
\hline & Shift work & $2.2 \%(2)$ \\
\hline \multirow{3}{*}{$\begin{array}{l}\text { Breadwinner } \\
\text { status }\end{array}$} & Sole & $34.8 \%(32)$ \\
\hline & Shared & $45.7 \%(42)$ \\
\hline & Partner & $19.6 \%(18)$ \\
\hline
\end{tabular}

was maintained at 18 months follow-up (83\%). Median time to RTW was 292 days (95\% CI 259 to 325 days) in this study population.

\section{Perceptions regarding work, work ability and work} limitations

Data on different perceptions about work are shown in table 2. There was a significant increase in the perceived

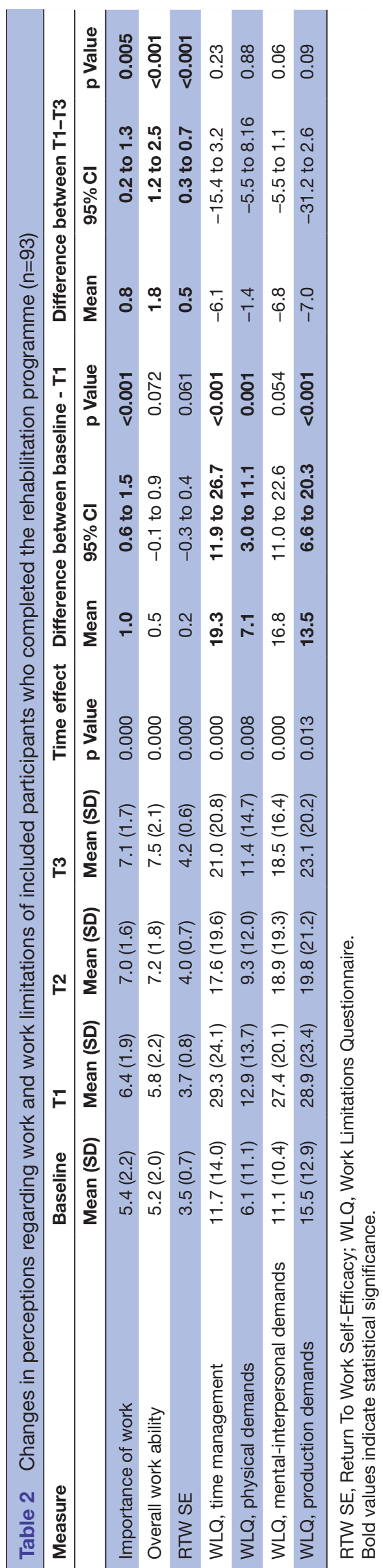


importance of work over time $(\mathrm{p}<0.001)$; the rating of 5.4 at baseline significantly improved to 6.4 at $\mathrm{T} 1$ and showed further improvement to 7.1 at T3.

The overall work ability $(p<0.001)$ and self-efficacy regarding RTW $(p<0.001)$ also showed a significant effect over time. Both the scores were not significantly better at T1 compared with baseline. At 18 months follow-up (T3) the participants showed a significant increase as compared with baseline in both overall WAI (difference was $1.8,95 \%$ CI 1.2 to 2.5 ) and self-efficacy (difference was $0.5,95 \%$ CI 0.3 to 0.7 ).

Perceived work limitations could only be assessed in those participants reported to have returned to work. Overall, a significant effect of time was seen in all WLQ subscales. Post hoc analysis showed that there was a significant increase in the percentage of time workers experienced limitations in meeting job demands regarding time management (19.3 at T1), physical tasks (7.1 at T1), and production (13.5 at T1). Although scores were lower when measured at $\mathrm{T} 3$, the reductions in perceived work limitations, of $-6,-1$ and -7 , respectively, were not significant.

\section{Fatigue and physical activity level}

Participants showed reducing fatigue levels over time $(\mathrm{p}<0.001)$, with a change in total score of -4.8 after programme completion (T1) and -8.3 at T3 on a scale of 20-100 (table 3). Considering the different subscales, scores at $\mathrm{T} 1$ show significantly less physical $(-1.6,95 \%$ CI -2.6 to -0.6$)$ and general fatigue $(-1.3,95 \% \mathrm{CI}-2.2$ to $-0.3)$, less reduced (improved) activity $(-1.6,95 \%$ CI -2.6 to -0.6$)$, and less reduced (improved) motivation $(-1.1$, $95 \%$ CI -1.9 to -0.3$)$. At T3 scores of all subscales were reduced further compared with fatigue levels obtained at T1 (physical fatigue $-1.9,95 \%$ CI -3.2 .to -0.5 ; general fatigue $-1.5,95 \% \mathrm{CI}-2.7$ to -0.2 ; reduced activity -2.0 , $95 \% \mathrm{CI}-3.3$ to -0.7 ; and reduced motivation $-0.9,95 \% \mathrm{CI}$ -1.7 to -0.1$)$.

Physical activity levels, measured in average hours a week performing moderate activity in work, leisure time, household and sports significantly changed over time. At T1 there was a significant reduction in activity level; $27 \pm 22$ hours versus $40 \pm 13$ hours of physical activity at baseline. Activity level was regained at T3, as this timeframe showed a significant increase from 13.8 hours to $38 \pm 16$ hours per week.

\section{Cardiorespiratory fitness and muscle strength}

Cardiorespiratory fitness as well as muscle strength were measured at the start and completion of the physical exercise programme. Paired t-tests results showed that there was a significant improvement of muscle strength (table 4). After training, all 1-RM results increased significantly, ranging from an improvement of $10 \mathrm{~kg}(95 \% \mathrm{CI}$ 8 to $12 \mathrm{~kg}$ ) for the deltiod pulley to an improvement of $45 \mathrm{~kg}$ (95\% CI 38 to $52 \mathrm{~kg}$ ) obtained for leg press.

At baseline, cardiorespiratory fitness measured as $\mathrm{VO}_{2}$ peak was $28.0 \pm 7.1 \mathrm{~mL} / \mathrm{min} / \mathrm{kg}$ on average. After the

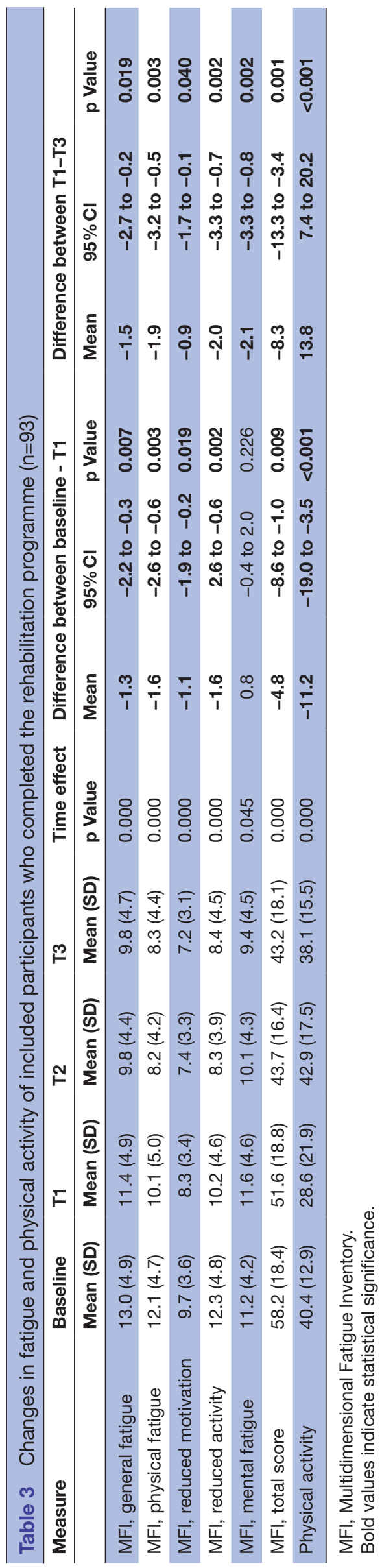


Table 4 Changes in muscle strength and cardiorespiratory fitness of included participants who completed the rehabilitation programme $(n=93)$

\begin{tabular}{lcclcc}
\hline Measure & $\begin{array}{l}\text { Mean } \\
\text { (SD) baseline }\end{array}$ & $\begin{array}{l}\text { Mean (SD) after } \\
\text { completion }\end{array}$ & Mean difference & 95\% Cl & p Value \\
\hline $\mathrm{VO}_{2}$ peak $(\mathrm{ml} / \mathrm{min} / \mathrm{kg})$ & $28.0(7.1)$ & $26.6(7.2)$ & -1.9 & -2.9 to -0.9 & $<0.001$ \\
\hline Maximal workload $(\mathrm{W})$ & $171.1(43.1)$ & $173.6(50.3)$ & 0.8 & -4.9 to 6.6 & 0.771 \\
\hline 1RM leg press $(\mathrm{kg})$ & $141.9(42.0)$ & $189.2(49.0)$ & 44.8 & 38.0 to 51.6 & $<0.001$ \\
1RM deltiod pulley $(\mathrm{kg})$ & $21.0(10.3)$ & $31.1(12.6)$ & 9.9 & 8.1 to 11.7 & $<0.001$ \\
$\begin{array}{l}\text { Maximal short exercise capacity } \\
\text { (steep ramp test) (W) }\end{array}$ & $190.1(53.7)$ & $220.1(51.8)$ & 27.5 & 20.6 to 34.4 & $<0.001$ \\
\hline
\end{tabular}

training programme this value was significantly decreased to an average of $26.6 \pm 7.2 \mathrm{~mL} / \mathrm{min} / \mathrm{kg}$. Maximal workload obtained in this $\mathrm{VO}_{2}$ peak test at baseline of $171 \pm 43 \mathrm{~W}$ did not change significantly $(173 \pm 50 \mathrm{~W})$ after programme completion). The maximal short exercise capacity, obtained with the steep ramp test, was significantly increased after completing the training programme from $190 \pm 54 \mathrm{~W}$ to $220 \pm 52 \mathrm{~W}$.

\section{Health-related quality of life}

HRQoL was measured multidimensionally and included global health, physical, role, cognitive, emotional and role functioning as well as symptoms such as fatigue, pain and nausea, which are presented in table 5. All these HRQoL scales showed a significant change over time, except for cognitive functioning $(\mathrm{p}=0.108)$ and pain $(p=0.055)$. Fatigue scores decreased over the total timeframe as the score at T1 (mean score 32 \pm 22 ) and at T3 (mean score $24 \pm 21$ ) were significantly lower than at baseline (mean score $43 \pm 25$ ) and $\mathrm{T} 1$, respectively. A similar pattern was seen for role functioning which continued to increase significantly between follow-up measurements from $60 \pm 28$ at baseline to $73 \pm 25$ at T1 and $85 \pm 26$ at T3. Physical functioning (mean difference 4, 95\% CI 1 to 7 ), social functioning (mean difference 12, 95\% CI 6 to 18) and global health status (mean difference $9,95 \%$ CI 5 to 13) showed an increase between $\mathrm{T} 1$ and $\mathrm{T} 3$, whereas the nausea scale showed a significant reduction in symptoms between baseline and $\mathrm{T} 1$ (mean difference $-6,95 \% \mathrm{CI}$ -12 to -1$)$.

\section{DISCUSSION}

Six months after the start of a multidisciplinary rehabilitation programme that combined occupational counselling with a supervised physical exercise programme, $59 \%$ of the cancer patients returned to work, $86 \%$ at 12 months and $83 \%$ at 18 months. In addition, significant improvements in the importance of work, work ability, RTW self-efficacy, muscle strength and quality of life were observed, whereas fatigue levels were significantly reduced.

\section{Interpretation of the findings}

The RTW rates found in this study were higher as compared with those found in the review of the literature of Mehnert. ${ }^{7}$ This pattern was shown at all three follow-up moments: at 6 months follow-up 59\% versus an average of $40 \%$ in the literature, at 12 months follow-up $86 \%$ versus $62 \%$, and at 18 months follow-up $83 \%$ versus $73 \%$. As these findings were based on 64 different studies, we cannot assess whether the baseline characteristics of our study population is representative of those reviewed by Mehnert. ${ }^{7}$ However, we do know that our participants are representative of the Dutch workforce in terms of working hours, type of contract and company size. We did have, nevertheless, an overrepresentation of highly educated women caused by the inclusion of a high percentage of breast cancer patients. Hence we also compared RTW rates to those of Dutch breast cancer patients only. Most recent findings were $70-71 \%$ for partial RTW 12 and 24 months after diagnosis, ${ }^{25} 26$ which are lower than our findings as well.

The recent controlled effect study from van Waart et $a l,{ }^{14}$ which was aimed at cancer patients undergoing chemotherapy as well and focused on RTW as secondary outcome, showed similar RTW rates compared with the current study in both intervention groups. RTW was assessed as yes or no, regardlessof the number of hours at the end of follow-up, which was 6 months after the end of chemotherapy. The RTW rate observed in their control group was $61 \%$. After receiving a home-based, low-intensity, self-managed physical activity programme the RTW was $79 \%$. After a moderate- to high-exercise programme supervised by a physical therapist the RTW was $83 \%$. However, in the study by van Waart et al the home-based exercises were repeated on a daily basis, while in our study the moderate exercise programme was performed twice a week for an average of 4.5 months. On the other hand, our study included the counselling of an occupational physician specialised in cancer-related problems with RTW, which is expected to have a positive effect on RTW. Both interventions produced good RTW rates, but because our current study was not a controlled study it is not possible to draw strong conclusions on the effect of the addition of occupational counselling.

The results show that work became increasingly important to the cancer patients in our study during and after completion of the intervention. Their perceived work ability and perceived self-efficacy with respect to work increased over the course of the intervention and during the year of follow-up as well. Interestingly, the 


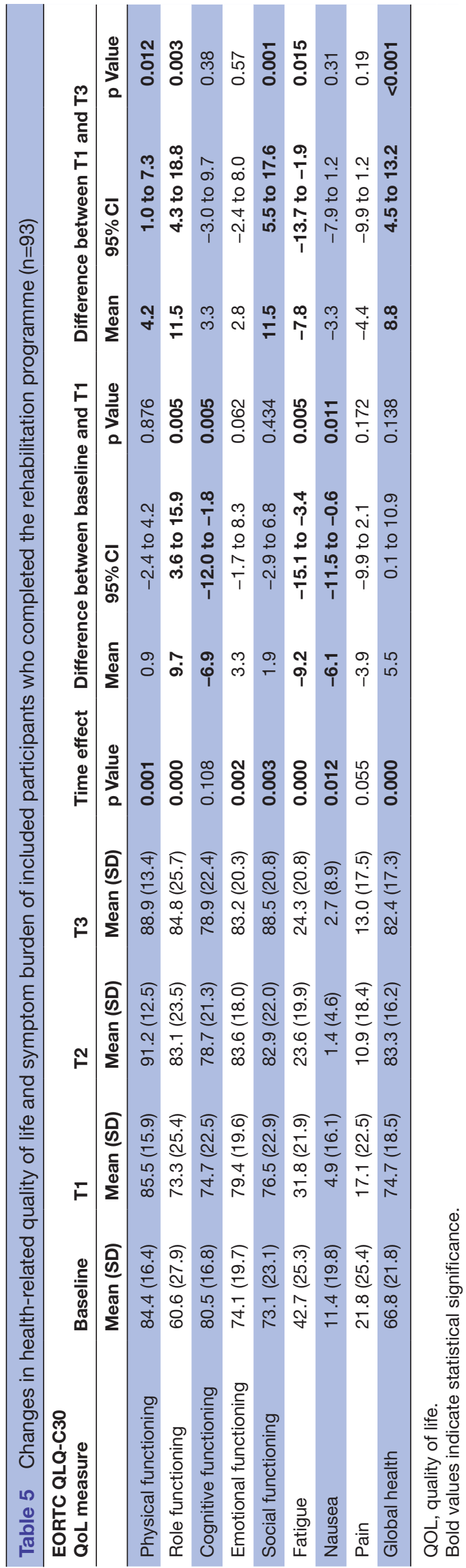

experienced importance of work of cancer patients in our study increased first, during the intervention and shortly after, while perceived work ability and self-efficacy for RTW showed larger improvements later, during the year of follow-up. A possible explanation could be that during the intervention, the consultations with the OOP might have affected the patient's perceived importance of work. At the same time, the adverse effects from the treatments including chemotherapy would have been present and recovery would still have been in progress, which could have negatively affected the patients' physical and cognitive work abilities and self-efficacy for RTW. An earlier study by Tamminga and colleagues also showed that during the initial RTW phase, physical or psychological side-effects hampered work resumption. ${ }^{35}$ After the intervention and during the year of follow-up, the effects of the chemotherapy would have ceased and thus the perceived work ability and self-efficacy for RTW improved.

In the current study participants showed decreased physical activity levels during and after chemotherapy which were regained through time while fatigue levels diminished over time. This could be an effect of time because the treatment with chemotherapy was coming to an end. However, Van Waart et al showed that moderateto high-intensity exercise, during which patients were receiving chemotherapy, had a beneficial effect on cardiorespiratory fitness as well as fatigue. ${ }^{14}$ Courneya et al found a significant improvement in fatigue among breast cancer patients who received aerobic exercise training or resistance exercise training during chemotherapy, compared with patients who did not receive any training. ${ }^{36}$ Based on the outcomes of the present study, as well the outcomes of the studies of van Waart et al and Courneya et al, physical rehabilitation interventions seem to reduce fatigue during chemotherapy.

The results of the present study imply that muscle strength had increased after completion of the programme. There was no increase in $\mathrm{VO}_{2}$ peak as was expected based on the studies of Backer $e t$ al and van Waart et al. ${ }^{12}{ }^{14}$ However, the training programme in this study was aimed more at improving muscle strength than on aerobic exercise because that is an important first step in (work) functioning. This could be an explanation for the lack of effect on $\mathrm{VO}_{2}$ peak.

HRQoL significantly increased on all domains except on the domains of cognitive functioning and pain. Several mechanisms might explain this increase. First, after the end of treatment and during follow-up, improvement of HRQoL EORTC scores is shown in most randomised controlled trials (RCTs) involving cancer patients ${ }^{37}$ and it is likely that the same course applied to the patients in our study. Second, at follow-up the large majority of our patients had returned to their work. Earlier studies have shown that RTW is positively related to HRQOL ${ }^{38}$ and therefore it is plausible that the patients in our study showed improved quality of life scores because they had returned to work. 


\section{Strengths and limitations}

An important strength of the present study is that to our knowledge it is the first to combine a physical training programme with occupational counselling in a multidisciplinary rehabilitation programme. Thereby RTW of cancer patients was supported through multiple strategies which were shown to be effective on RTW in a Cochrane review. ${ }^{11}$ The training programme was supervised by trained physiotherapists and sports physicians. Validated measures were utilised to assess fitness and muscle strength and validated questionnaires were used to assess work ability, work limitations, self-efficacy, fatigue, and quality of life. As a result, the results obtained in this study are reliable. However, there are also limitations to this study. In the first place, we did not randomise patients because we based the study on a real life situation and patients who met the inclusion criteria were invited to participate. Because of the lack of a control group, there is no direct proof of the effects of our multidisciplinary programme. However, the controlled effect studies showed to a large extent comparable findings. ${ }^{14} 1636$ Another limitation of our study design is the possible bias in the selection of participants with respect to the type of cancer and the motivation to participate in a physical exercise programme. We mainly included female patients with breast cancer with a relatively high level of education and we cannot therefore be sure whether the findings can be applied to male patients, people with lower levels of education, and patients with other types of cancer. Furthermore, the cancer patients who were included were highly motivated to participate. Those who did not participate might have been less motivated and more likely to have a different lifestyle. At the same time the intervention might have been most beneficial for the latter group but we could not reach them. It is also not yet clear whether this type of intervention should be offered to all cancer patients or whether it is better to 'personalise' the intervention to certain subgroups of patients.

Based on the present study, it is recommended to investigate further the effects of this multidisciplinary intervention, including occupational counselling and an exercise programme, in a controlled study. An implication for research is to study the added value of an OOP in an RCT and in other hospitals in order to assess the feasibility of the intervention more broadly and to identify subgroups of patients in need of differentiated approaches.

\section{Conclusion}

A multidisciplinary rehabilitation programme, combining specialised occupational counselling with a physical exercise programme supervised by a sports physician and a physiotherapist, is likely to support RTW among cancer patients. The programme also seems to improve self-reported fatigue levels and physical activity, perceived importance of work, work ability, RTW, self-efficacy, quality of life, muscle strength and work perceptions. It can be considered a promising intervention to support cancer patients in resuming their work.

\section{Author affiliations}

${ }^{1}$ Coronel Institute of Occupational Health, Academic Medical Center, University of Amsterdam, Amsterdam Public Health research institute, Amsterdam, The Netherlands

${ }^{2}$ Rijnlands Rehabilitation Center, Leiden, The Netherlands

${ }^{3}$ Leiden University Medical Center, Leiden, The Netherlands

${ }^{4}$ Department of Sports Medicine, Haaglanden Medical Center, The Hague, The Netherlands

${ }^{5}$ Department of Sports Medicine, Isala Medical Center, Zwolle, The Netherlands ${ }^{6}$ University Twente, Enschede, The Netherlands

${ }^{7}$ Rijnstate Hospital, Arnhem, The Netherlands

${ }^{8}$ The Netherlands Cancer Institute, Amsterdam, The Netherlands

Correction notice This paper has been amended since it was published Online First. Owing to a scripting error, some of the publisher names in the references were replaced with 'BMJ Publishing Group'. This only affected the full text version, not the PDF. We have since corrected theseerrors and the correct publishers have been inserted into the references.

Contributors ML: acquisition, analysis, and interpretation of data for the work; drafting the work; final approval of the version to be published; agreement to be accountable for all aspects of the work in ensuring that questions related to the accuracy or integrity of any part of the work are appropriately investigated and resolved. IG: acquisition, analysis, and interpretation of data for the work; revising the work critically for important intellectual content; final approval of the version to be published; agreement to be accountable for all aspects of the work in ensuring that questions relating to the accuracy or integrity of any part of the work are appropriately investigated and resolved. $\mathrm{IH}$ : acquisition, analysis, and interpretation of data for the work; drafting and revising the work critically for important intellectual content; final approval of the version to be published; agreement to be accountable for all aspects of the work in ensuring that questions relating to the accuracy or integrity of any part of the work are appropriately investigated and resolved. TR: conception or design of the work and acquisition of data for the work; revising the work critically for important intellectual content; final approval of the version to be published; agreement to be accountable for all aspects of the work in ensuring that questions relating to the accuracy or integrity of any part of the work are appropriately investigated and resolved. PV: acquisition, analysis, and interpretation of data for the work; revising the work critically for important intellectual content; final approval of the version to be published; agreement to be accountable for all aspects of the work in ensuring that questions relating to the accuracy or integrity of any part of the work are appropriately investigated and resolved. SB: acquisition, analysis, and interpretation of data for the work; revising the work critically for important intellectual content; final approval of the version to be published; agreement to be accountable for all aspects of the work in ensuring that questions relating to the accuracy or integrity of any part of the work are appropriately investigated and resolved. AS: acquisition, analysis, and interpretation of data for the work; revising the work critically for important intellectual content; final approval of the version to be published; agreement to be accountable for all aspects of the work in ensuring that questions relating to the accuracy or integrity of any part of the work are appropriately investigated and resolved. WH: conception and design of the work; revising the work critically for important intellectual content; final approval of the version to be published; agreement to be accountable for all aspects of the work in ensuring that questions relating to the accuracy or integrity of any part of the work are appropriately investigated and resolved. MF: conception and design of the work; analysis, and interpretation of data for the work; revising the work critically for important intellectual content; final approval of the version to be published; agreement to be accountable for all aspects of the work in ensuring that questions relating to the accuracy or integrity of any part of the work are appropriately investigated and resolved. $A B$ : conception and design of the work; acquisition, analysis, and interpretation of data for the work; drafting the work and revising it critically for important intellectual content; final approval of the version to be published; agreement to be accountable for all aspects of the work in ensuring that questions relating to the accuracy or integrity of any part of the work are appropriately investigated and resolved.

Funding The study was funded by Alpe d'Huzes Foundation / Dutch Cancer Society. Grant number: NKI 2010-4854.

Competing interests None declared. 
Ethics approval AMC medical ethics committees.

Provenance and peer review Not commissioned; externally peer reviewed. Data sharing statement № unpublished data from the study are available.

Open Access This is an Open Access article distributed in accordance with the Creative Commons Attribution Non Commercial (CC BY-NC 4.0) license, which permits others to distribute, remix, adapt, build upon this work non-commercially, and license their derivative works on different terms, provided the original work is properly cited and the use is non-commercial. See: http://creativecommons.org/ licenses/by-nc/4.0/

(C) Article author(s) (or their employer(s) unless otherwise stated in the text of the article) 2017. All rights reserved. No commercial use is permitted unless otherwise expressly granted.

\section{REFERENCES}

1. Petersson LM, Wennman-Larsen A, Nilsson M, et al. Work situation and sickness absence in the initial period after breast cancer surgery. Acta Oncol 2011;50:282-8.

2. Nachreiner NM, Ghebre RG, Virnig BA, et al. Early work patterns for gynaecological cancer survivors in the USA. Occup Med 2012;62:23-8.

3. Saunders SL, Nedelec B. What work means to people with work disability: a scoping review. J Occup Rehabil 2014;24:100-10.

4. Stergiou-Kita M, Grigorovich A, Tseung V, et al. Qualitative metasynthesis of survivors' work experiences and the development of strategies to facilitate return to work. J Cancer Surviv 2014;8:657-70.

5. Isaksson J, Wilms T, Laurell G, et al. Meaning of work and the process of returning after head and neck cancer. Support Care Cancer 2016;24:205-13.

6. Banning M. Employment and breast cancer: a meta-ethnography. Eur J Cancer Care 2011;20:708-19.

7. Mehnert A. Employment and work-related issues in cancer survivors. Crit Rev Oncol Hematol 2011;77:109-30.

8. de Boer AG, Taskila T, Ojajärvi A, et al. Cancer survivors and unemployment: a meta-analysis and meta-regression. JAMA 2009;301:753-62.

9. Lee MK, Yun YH. Working situation of cancer survivors versus the general population. J Cancer Surviv 2015;9:349-60.

10. Silver JK, Baima J, Newman R, et al. Cancer rehabilitation may improve function in survivors and decrease the economic burden of cancer to individuals and society. Work 2013;46:455-72.

11. de Boer AG, Taskila T, Tamminga SJ, et al. Interventions to enhance return-to-work for cancer patients. Cochrane Db Syst Rev 2015;25:CD007569.

12. De Backer IC, Vreugdenhil G, Nijziel MR, et al. Long-term follow-up after cancer rehabilitation using high-intensity resistance training: persistent improvement of physical performance and quality of life. Br J Cancer 2008;99:30-6.

13. Mishra SI, Scherer RW, Snyder C, et al. The effectiveness of exercise interventions for improving health-related quality of life from diagnosis through active cancer treatment. Oncol Nurs Forum 2015;42:E33-53.

14. van Waart $\mathrm{H}$, Stuiver MM, van Harten WH, et al. Effect of lowintensity physical activity and moderate- to high-intensity physical exercise during adjuvant chemotherapy on physical fitness, fatigue, and chemotherapy completion rates: results of the paces randomized clinical trial. J Clin Oncol 2015;33:1918-27.

15. Groeneveld IF, de Boer AG, Frings-Dresen MH. Physical exercise and return to work: cancer survivors' experiences. J Cancer Surviv 2013;7:237-46.

16. Thijs KM, de Boer AG, Vreugdenhil G, et al. Rehabilitation using highintensity physical training and long-term return-to-work in cancer survivors. J Occup Rehabil 2012;22:220-9.
17. Feuerstein M, Todd BL, Moskowitz MC, et al. Work in cancer survivors: a model for practice and research. J Cancer Surviv 2010;4:415-37

18. Mehnert A, de Boer A, Feuerstein M. Employment challenges for cancer survivors. Cancer 2013;119(Supp 11):2151-9.

19. Verbeek J, Spelten E, Kammeijer M, et al. Return to work of cancer survivors: a prospective cohort study into the quality of rehabilitation by occupational physicians. Occup Environ Med 2003:60:352-7.

20. Taskila T, Lindbohm ML. Factors affecting cancer survivors' employment and work ability. Acta Oncol 2007;46:446-51.

21. Groeneveld IF, de Boer AG, Frings-Dresen MH. A multidisciplinary intervention to facilitate return to work in cancer patients: intervention protocol and design of a feasibility study. BMJ Open 2012;2:e001321.

22. Heijbel B, Josephson M, Jensen I, et al. Return to work expectation predicts work in chronic musculoskeletal and behavioral health disorders: prospective study with clinical implications. J Occup Rehabil 2006;16:169-80.

23. Ekbladh E, Thorell LH, Haglund L. Perceptions of the work environment among people with experience of long term sick leave. Work 2010;35:125-36.

24. de Boer AG, Verbeek JH, Spelten ER, et al. Work ability and returnto-work in cancer patients. Br J Cancer 2008;98:1342-7.

25. Roelen CA, Koopmans PC, Schellart AJ, et al. Resuming work after cancer: a prospective study of occupational register data. J Occup Rehabil 2011:21:431-40.

26. Roelen CA, Koopmans PC, Groothoff JW, et al. Return to work after cancer diagnosed in 2002, 2005 and 2008. J Occup Rehabil 2011;21:335-41.

27. De Backer IC, Schep G, Hoogeveen A, et al. Exercise testing and training in a cancer rehabilitation program: the advantage of the steep ramp test. Arch Phys Med Rehabil 2007;88:610-6.

28. Tuomi K, Ilmarinen J, Jahkola A. Work ability index. Helsinki: Finnish Institute of Occupational Health. 2nd revised ed, 1998.

29. Lagerveld SE, Blonk RWB, Brenninkmeijer V, et al. Return to work among employees with mental health problems: development and validation of a self-efficacy questionnaire. Work Stress 2010;24:359-75.

30. Lerner D, Amick BC, Rogers WH, et al. The work limitations questionnaire. Med Care 2001;39:72-85.

31. De Backer IC, Schep G, Backx FJ, et al. Resistance training in cancer survivors: a systematic review. Int J Sports Med 2009;30:703-12.

32. Smets EM, Garssen B, Bonke B, et al. The Multidimensional Fatigue Inventory (MFI) psychometric qualities of an instrument to assess fatigue. J Psychosom Res 1995;39:315-25.

33. Wendel-Vos GC, Schuit AJ, Saris WH, et al. Reproducibility and relative validity of the short questionnaire to assess health-enhancing physical activity. J Clin Epidemiol 2003;56:1163-9.

34. Aaronson NK, Ahmedzai S, Bergman B, et al. The European Organization for Research and Treatment of Cancer QLQ-C30: a quality-of-life instrument for use in international clinical trials in oncology. J Natl Cancer Inst 1993;85:365-76.

35. Tamminga SJ, de Boer AG, Verbeek JH, et al. Breast cancer survivors' views of factors that influence the return-to-work process-a qualitative study. Scand J Work Environ Health 2012;38:144-54

36. Courneya KS, Segal RJ, Mackey JR, et al. Effects of aerobic and resistance exercise in breast cancer patients receiving adjuvant chemotherapy: a multicenter randomized controlled trial. J Clin Oncol 2007;25:4396-404.

37. Zikos E, Coens C, Quinten C, et al. The added value of analyzing pooled health-related quality of life data: a review of the EORTC PROBE initiative. J Natl Cancer Inst 2016;108:pii: djv391.

38. Mellon S, Northouse LL, Weiss LK. A population-based study of the quality of life of cancer survivors and their family caregivers. Cancer Nurs 2006;29:120-31. 